Stochastics and Statistics

\title{
The role of repair strategy in warranty cost minimization: An investigation via quasi-renewal processes
}

\author{
Gülay Samatlı-Paç ${ }^{\mathrm{a}}$, Mehmet R. Taner ${ }^{\mathrm{b}, *}$ \\ ${ }^{a}$ Department of Decision Sciences, LeBow College of Business, Drexel University, Philadelphia, PA, USA \\ ${ }^{\mathrm{b}}$ Department of Industrial Engineering, Bilkent University, Ankara, Turkey
}

\section{A R T I C L E I N F O}

\section{Article history:}

Received 24 August 2007

Accepted 30 June 2008

Available online 17 July 2008

\section{Keywords:}

Reliability

Imperfect repair

Quasi-renewal processes

Two-dimensional warranty

Warranty cost

\begin{abstract}
A B S T R A C T
Most companies seek efficient rectification strategies to keep their warranty related costs under control. This study develops and investigates different repair strategies for one- and two-dimensional warranties with the objective of minimizing manufacturer's expected warranty cost. Static, improved and dynamic repair strategies are proposed and analyzed under different warranty structures. Numerical experimentation with representative cost functions indicates that performance of the policies depend on various factors such as product reliability, structure of the cost function and type of the warranty contract.
\end{abstract}

(c) 2008 Elsevier B.V. All rights reserved.

\section{Introduction}

Extensive warranties are commonly offered by a wide range of manufacturers as a means of survival in increasingly fierce market conditions. Faced with the challenge of keeping the associated costs under control, most companies seek efficient rectification strategies. In this study, different repair strategies are developed and investigated under one- and two-dimensional warranties with the intent of minimizing the manufacturer's expected warranty cost. Quasi-renewal processes are used to model the product failures along with the associated repair actions. Based on quasi-renewal processes, three different repair policies - static, improved and dynamic - are proposed, and representative cost functions are developed to evaluate the effectiveness of these alternative policies.

In a one-dimensional warranty, the warrantor agrees to rectify or compensate the customer for the failed items within a certain time limit after time of sale. A two-dimensional warranty is a natural extension where the warranty period is characterized by a region defined simultaneously by time and usage. Examples of twodimensional warranties are widely seen in the automotive industry where vehicles are covered under warranty until a certain age or mileage after the initial purchase.

Karim and Suzuki (2005) provide a recent survey of the literature on statistical models and methods for warranty analysis. They present a summary of important mathematical findings such as

\footnotetext{
* Corresponding author. Tel.: +90 312 2901264; fax: +90 3122664054

E-mail address: mrtaner@bilkent.edu.tr (M.R. Taner).
}

estimators of critical parameters used in the analysis of warranty claim data. Thomas and Rao (1999) and Murthy and Djamaludin (2002) are also important review papers on product warranty. Thomas and Rao (1999) adopt a management perspective and focus on the works that address quantification of warranty costs and determination of warranty policies. They also present some research directions. Murthy and Djamaludin (2002) follow a broader perspective. They build on Murthy and Blischke's (1992a,b) paper and cover the pertinent academic developments in the areas of cost analysis, engineering design, marketing, logistics and management systems. They also mention applications in some other related areas such as law, accounting, economics and sociology.

Of particular interest for the current study is the modeling of rectification actions in the warranty context. Majority of the literature on one- and two-dimensional warranties considers perfect and minimal repairs. Imperfect repair is widely modeled as a combination of perfect and minimal repair. Barlow and Hunter (1960) are the first to combine the perfect and minimal repair under onedimensional warranties. The studies of Cleroux et al. (1979), Boland and Proschan (1982), Phelps (1983) and Nguyen and Murthy (1984) give some other examples of combination repair/replace models under one-dimensional warranty. Choi and Yun (2006) investigate the performance of several functions to calculate a threshold limit on the acceptable cost of minimum repair. Their model replaces the failed product if the expected cost of minimum repair exceeds the predetermined threshold. Iskandar and Murthy (2003), Iskandar et al. (2005), Chukova and Johnston (2006) and Chukova et al. (2006) apply the combination type imperfect repair models in the context of two-dimensional warranties. In these four 
papers, warranty region is divided in various ways into disjoint sub-regions with a priory decision on whether to pursue minimum or complete repair within each region. The objective is to determine the sub-regions so as to minimize the expected warranty cost.

An alternative approach is a generalization of the renewal process in which the product failure characteristics are revised after each failure as in the virtual age model proposed in Kijima (1989). In this model, the virtual age of the failed product is adjusted by a factor that reflects the degree of repair so as to bring it to a desired state somewhere between as good as new and as bad as old. Yanez et al. (2002) propose the use of Bayesian and maximum likelihood methods to estimate the model parameters for the generalized renewal process. Dagpunar (1997) and Dimitrov et al. (2004) use modified versions of the virtual age model.

Wang and Pham (1996a,b) and Bai and Pham (2005) use a further alternative and model the imperfect repairs in a single-dimensional warranty context as a quasi-renewal process. In the current paper, we extend their methodology to multi-dimensional warranties and adopt the appropriate version in both one and two-dimensional analyses. Due to the significance of the chronological age in warranty applications, quasi-renewal processes have greater intuitive appeal than the virtual age models in a warranty context. Quasi-renewal processes yield a mathematically convenient approach to calculate the number of failures within the warranty period.

The remainder of the paper is organized as follows. Section 2 presents a detailed description of the problem. Section 3 describes the methodology used to model the failure and repair process, defines a representative cost function, and develops different repair strategies. Renewal equations are also characterized in this section to calculate the expected number of failures under different types of two-dimensional warranties. Section 4 presents an application of the proposed approach in a real life industrial example. The approach is investigated under a variety of settings through computational experimentation in Section 5. Section 6 concludes the paper and offers some suggested directions for future research.

\section{Problem description}

The objective is to investigate the performance of alternative repair strategies in terms of the manufacturer's expected warranty cost under one- and two-dimensional warranties. The repair strategy has an effect on both the cost of a single repair and the number of repairs to be covered under warranty. Evidently, the total expected warranty cost is also a function of various other parameters such as product's reliability characteristics, the type of the warranty contract and the mathematical structure of the cost function. Before we introduce the detailed scheme within which we control these parameters and pursue our analyses, we make a few simplifying assumptions.

We first assume that buyers of a given product have similar usage patterns. Thus, the time until failure follows the same probability distribution. Next we assume that all claims made during the warranty period are valid and hence must be properly rectified by the manufacturer in accordance with the terms of the warranty contract. Finally, we consider the repair duration to be significantly smaller than the length of the warranty period so that repairs can be modeled to occur instantaneously.

With respect to repair actions, we study imperfect repairs based on a quasi-renewal process. The quasi-renewal process is characterized by a scaling parameter that alters the random variable corresponding to time until next failure after each renewal. In other words, this parameter indicates the degree of deterioration or improvement. For example, if the scaling parameter is between 0 and 1 , it indicates deterioration; whereas if it is greater than 1 , it indicates an improvement. Hereon, we refer to this parameter as the degree of repair. The degree of repair also determines the amount of change in the mean inter-failure time and the failure rate before and after the renewal.

To compare various policies, we use the expected total cost over the warranty period. Representative cost functions that address this issue for one- and two-dimensional warranties are proposed in Section 3.2.

\section{Modeling the failure and repair process}

In this part, we first present in Section 3.1, the multiple quasirenewal processes to model the failure and associated repair process. Then in Section 3.2, representative cost functions for oneand two-dimensional warranties are introduced. In Section 3.3, different repair strategies are proposed. Lastly, calculation of the expected number of failures under one- and two-dimensional warranties is discussed in Section 3.4.

\subsection{Multiple quasi-renewal process}

In this section, the univariate quasi-renewal processes proposed by Wang and Pham (1996b) are generalized to multivariate distributions to model $n$-dimensional warranties. For a failure process defined along $n$-dimensions, let $\mathbf{X}_{i}=\left(X_{1 i}, X_{2 i}, \ldots, X_{n i}\right), i=1,2,3, \ldots$ represent an $n$-dimensional random vector where $X_{k i}$ denotes the length of the interval between the $(i-1)$ th and ith successive renewals on the $k$ th dimension with $X_{k 0}=0$ for $k=1,2, \ldots, n$. Consider a counting process $\left\{N\left(x_{1}, x_{2}, \ldots, x_{n}\right) ; x_{k}>0, k=1, \ldots, n\right\}$ that represents the number of events in region $(0,0, \ldots, 0) \times\left(x_{1}, x_{2}, \ldots, x_{n}\right)$. This process is an $n$-dimensional quasirenewal process if

$\left[\begin{array}{l}X_{1 i} \\ \vdots \\ X_{n i}\end{array}\right]=\left[\begin{array}{lll}\alpha_{1}^{i-1} & \cdots & 0 \\ \vdots & \ddots & \vdots \\ 0 & \cdots & \alpha_{n}^{i-1}\end{array}\right]\left[\begin{array}{l}Y_{1 i} \\ \vdots \\ Y_{n i}\end{array}\right]=\left[\begin{array}{l}\alpha_{1}^{i-1} Y_{1 i} \\ \vdots \\ \alpha_{n}^{i-1} Y_{n i}\end{array}\right]$

where $\alpha_{k}$ is a positive real constant that measures the degree of repair in the $k$ th dimension for $k=1,2, \ldots, n$ and $\mathbf{Y}_{i}$ is an $n$-dimensional i.i.d. random vector for all $i$.

Let $F\left(y_{1 i}, y_{2 i}, \ldots, y_{n i}\right)$ and $f\left(y_{1 i}, y_{2 i}, \ldots, y_{n i}\right)$ be the c.d.f. and p.d.f. of $\mathbf{Y}_{i}=\left(Y_{1 i}, Y_{2 i}, \ldots, Y_{n i}\right)$ for $i=1,2,3, \ldots$ respectively. Then the cumulative distribution and density functions of $\mathbf{X}_{i}$ can be written as follows:

$$
\begin{aligned}
& F_{i}\left(x_{1 i}, \ldots, x_{n i}\right)=F\left(\alpha_{1}^{1-i} x_{1 i}, \ldots, \alpha_{n}^{1-i} x_{n i}\right), \\
& f_{i}\left(x_{1 i}, \ldots, x_{n i}\right)=\frac{\partial^{n} F_{i}\left(x_{1 i}, \ldots, x_{n i}\right)}{\partial x_{1 i} \ldots \partial x_{n i}}=\prod_{k=1}^{n} \alpha_{k}^{1-i} f\left(\alpha_{1}^{1-i} x_{1 i}, \ldots, \alpha_{n}^{1-i} x_{n i}\right) .
\end{aligned}
$$

The probability function of $N\left(x_{1}, x_{2}, \ldots, x_{n}\right)$ can be derived by using the relationship $N\left(x_{1}, x_{2}, \ldots, x_{n}\right) \geqslant i \Leftrightarrow S_{i} \leqslant\left(x_{1}, x_{2}, \ldots, x_{n}\right)$, where $S_{i}$ is the occurrence point of the $i$ th event. The probability that there will be $i$ events within region $(0,0, \ldots, 0) \times\left(x_{1}, x_{2}, \ldots, x_{n}\right)$ is

$$
\begin{aligned}
& P\left(N\left(x_{1}, \ldots, x_{n}\right)=i\right)=P\left(S_{i} \leqslant\left(x_{1}, \ldots, x_{n}\right)\right)-P\left(S_{i+1} \leqslant\left(x_{1}, \ldots, x_{n}\right)\right), \\
& P\left(N\left(x_{1}, \ldots, x_{n}\right)=i\right)=F^{(i)}\left(x_{1}, \ldots, x_{n}\right)-F^{(i+1)}\left(x_{1}, \ldots, x_{n}\right) i=1,2, \ldots
\end{aligned}
$$

where $F^{(i)}$ is the $i$-fold convolution of $F$ with $F^{(0)}\left(x_{1}, x_{2}, \ldots, x_{n}\right)=1$.

Consequently, the renewal function for the $n$-dimensional quasi-renewal process is obtained as follows:

$$
\begin{aligned}
M_{q}^{n}\left(x_{1}, \ldots, x_{n}\right) & =E\left[N\left(x_{1}, \ldots, x_{n}\right)\right]=\sum_{k=0}^{\infty} k P\left(N\left(x_{1}, \ldots, x_{n}\right)=k\right) \\
& =\sum_{k=1}^{\infty} F^{(k)}\left(x_{1}, \ldots, x_{n}\right)
\end{aligned}
$$


This function is different from the ordinary renewal functions in that the renewal periods are not identically distributed.

For a product whose lifetime is characterized by a singledimension such as time to failure, let $T_{i}$ be the time interval between $(i-1)$ th and $i$ th failures with $T_{0}=0$. Then $N(t)$ would be a univariate quasi-renewal process characterized by

$T_{i}=\alpha^{i-1} Y_{i} \quad i=1,2,3, \ldots$

where $\alpha>0$ represents the degree of repair, and $Y_{i}$ 's are i.i.d. random variables with c.d.f. $F(y)$. Similarly for a product whose lifetime depends also on usage between failures, define $X_{i}$ as the usage between the $(i-1)$ th and $i$ th failures, with $X_{0}=0$. Then, the corresponding bivariate quasi-renewal process $N(t, x)$ would be characterized by

$$
\begin{aligned}
& T_{i}=\alpha_{1}^{i-1} Y_{i}, \quad i=1,2,3, \ldots \\
& X_{i}=\alpha_{2}^{i-1} Z_{i}, \quad
\end{aligned}
$$

where $\alpha_{1}$ and $\alpha_{2}$ are positive real constants representing the degree of repair on the respective dimension, and $\left(Y_{i}, Z_{i}\right)$ 's are i.i.d. random variables with c.d.f. $F(y, z)$.

\subsection{Modeling the warranty cost}

Majority of the warranty literature assumes a constant cost term throughout the entire warranty period. This term aggregates all related components such as the loss of goodwill, the cost of repairs and other transaction costs. In this study, we propose and use new cost functions that have fixed and variable components. The fixed component is paid independently of the degree of repair and represents the costs such as loss of goodwill, shipment or setup, whereas the variable cost includes direct labor and direct material costs and it increases in parallel with the degree of repair.

The following additional notation will be needed to define an appropriate cost function that displays these characteristics for an $n$-dimensional warranty:

$\mathbf{W}$ : Vector indicating the limit of the warranty region

$N(\mathbf{W})$ : Number of failures within the warranty region

$c$ : Fixed cost charged for each failure

$c_{k}$ : Variable unit cost in the $k$ th dimension

$C\left(\mathbf{W}, \alpha_{1}, \ldots, \alpha_{n}\right)=\left(c+c_{1} \alpha_{1}+\cdots+c_{n} \alpha_{n}\right) N(\mathbf{W})$.

The expected cost is then as follows:

$E\left[C\left(\mathbf{W}, \alpha_{1}, \ldots, \alpha_{n}\right)\right]=\left(c+c_{1} \alpha_{1}+\cdots+c_{n} \alpha_{n}\right) E[N(\mathbf{W})]$,

where $E[N(\mathbf{W})]$ is the expected number of failures within the warranty region.

\subsection{Modeling the repair action}

In this section, we propose three different imperfect repair policies that rely on the quasi-renewal process. These are the static, improved and dynamic policies for the one- and two-dimensional warranties.

\subsubsection{Static policies}

These policies rectify all breakdowns within the warranty period in the same manner. That is, $\alpha$ for one-dimensional, $\alpha_{1}$ and $\alpha_{2}$ for two-dimensional warranties are assumed constant over the warranty period. The degree of repair takes values between 0 and 1 where the degree of repair being equal to 1 corresponds to perfect repair. Although small $\alpha, \alpha_{1}$ and $\alpha_{2}$ values result in small unit costs, the total cost may be large due to a large expected number of fail- ures. Therefore, it is important for the manufacturer to find a trade-off in the degree of repair that minimizes the total cost.

\subsubsection{Improved policy}

In this policy, the product is replaced by an improved version after the first failure and in the succeeding failures it is repaired according to some static policy. The improved policy may be particularly suitable for high-tech products for which a newer, improved version of the product may become available by the first failure. Let $\beta$ be the degree of improvement between these two versions of the product. For one-dimensional warranties, the interfailure times under the improved policy can be modeled as follows:

$$
\begin{aligned}
& T_{1}=Y_{1}, \\
& T_{2}=\beta Y_{2}, \\
& T_{i}=\alpha^{i-2} \beta Y_{i} \quad \text { for } i \geqslant 3 .
\end{aligned}
$$

The corresponding total expected warranty cost over the warranty period $W$ is defined as follows:

$$
\begin{aligned}
E C^{(1, \mathrm{imp})}= & \left(c+\beta c_{1}\right)\left(F^{(1)}(W)-F^{(2)}(W)\right)+\sum_{i=2}^{\infty}\left[\left(c+\beta c_{1}\right)\right. \\
& \left.+(i-1)\left(c+\alpha c_{1}\right)\right]\left(F^{(i)}(W)-F^{(i+1)}(W)\right) \\
& =\left(c+\beta c_{1}\right) F^{(1)}(W)+\left(c+\alpha c_{1}\right)\left[E(N(W))-F^{(1)}(W)\right]
\end{aligned}
$$

For the two-dimensional warranties, define $\beta_{k}$ as the degree of improvement with respect to dimension $k$. Then, the improved policy for the two-dimensional warranties can be modeled as follows:

$$
\begin{aligned}
& T_{1}=Y_{1} \text {, } \\
& X_{1}=Z_{1} \text {, } \\
& T_{2}=\beta_{1} Y_{2} \text {, } \\
& \text { and } X_{2}=\beta_{2} Z_{2} \text {, } \\
& T_{i}=\alpha_{1}^{i-2} \beta_{1} Y_{i} \text { for } i \geqslant 3 \\
& X_{i}=\alpha_{2}^{i-2} \beta_{2} Z_{i} \text { for } i \geqslant 3 \text {. }
\end{aligned}
$$

The corresponding total expected warranty cost before time limit $W$ and usage limit $U$ is defined as follows:

$$
\begin{aligned}
E C^{(2, \text { imp })}= & \left(c+\beta_{1} c_{1}+\beta_{2} c_{2}\right)\left(F^{(1)}(W, U)-F^{(2)}(W, U)\right) \\
& +\sum_{i=2}^{\infty}\left[\left(c+\beta_{1} c_{1}+\beta_{2} c_{2}\right)\right. \\
& \left.+(i-1)\left(c+\alpha_{1} c_{1}+\alpha_{2} c_{2}\right)\right]\left(F^{(i)}(W, U)-F^{(i+1)}(W, U)\right) \\
& =\left(c+\beta_{1} c_{1}+\beta_{2} c_{2}\right) F^{(1)}(W, U)+\left(c+\alpha_{1} c_{1}+\alpha_{2} c_{2}\right) \\
& \times\left[E(N(W, U))-F^{(1)}(W, U)\right] .
\end{aligned}
$$

\subsubsection{Dynamic policy}

In this policy, the degree of repair changes as a decreasing function of time. The motivation is to decrease the expected cost while carrying the product to the end of the warranty period in an operational state. To model the failure time under the dynamic policy, we denote the degree of repair by $\alpha(t)$ in one-dimensional, and by $\alpha_{1}(t)$ and $\alpha_{2}(t)$ in the two-dimensional case to indicate that it is now a function of time. Since good repair becomes increasingly undesirable towards the end of warranty period, it is preferable for these functions to be concave.

The failure times and the total expected warranty in the univariate model are as follows:

$T_{1}=Y_{1}$,

$T_{i}=\alpha\left(\sum_{k=1}^{i-1} T_{k}\right) Y_{i}, \quad i \geqslant 2$,

$E C^{(1, \mathrm{dyn})}=\sum_{i=1}^{\infty}\left[c+c_{1} * E\left(\alpha\left(\sum_{k=1}^{i} T_{k}\right)\right)\right] F^{(i)}(W)$.

In the two-dimensional policies, we attempt to ensure that the repair is equally effective in both dimensions. To accomplish this, we con- 
sider rectification in proportion to the mean time $\left(\mu_{1}\right)$ and mean usage $\left(\mu_{2}\right)$ until the first failure in the corresponding dimension. That is, we enforce $\frac{\alpha_{1}(t)}{\alpha_{2}(t)}=\frac{\mu_{1}}{\mu_{2}}$ for all $t$ with the condition that $\alpha_{1}(t), \alpha_{2}(t) \leqslant 1$. Mathematically, the process is characterized by the following:

$$
\begin{aligned}
& T_{1}=Y_{1} \text {, } \\
& T_{i}=\alpha_{1}\left(\sum_{k=1}^{i-1} T_{k}\right) Y_{i}, \quad i \geqslant 2 \quad \text { and } \quad \begin{array}{l}
X_{1}=Z_{1}, \\
X_{i}=\alpha_{2, i} Z_{i}, \quad i \geqslant 2,
\end{array}
\end{aligned}
$$

where $\alpha_{2, i}$ is the degree of repair for the usage dimension such that $\frac{\alpha_{1}\left(\sum_{k=1}^{i-1} T_{k}\right)}{\mu_{1}}=\frac{\alpha_{2, i}}{\mu_{2}} \Rightarrow \alpha_{2, i}=\alpha_{1}\left(\sum_{k=1}^{i-1} T_{k}\right) \frac{\mu_{2}}{\mu_{1}}$. Consequently, the total expected cost is as follows:

$E C^{(2, \mathrm{dyn})}=\sum_{i=1}^{\infty}\left[c+\left(c_{1}+c_{2} \frac{\mu_{2}}{\mu_{1}}\right) * E\left(\alpha\left(\sum_{k=1}^{i} T_{k}\right)\right)\right] F^{(i)}(W)$

In a dynamic policy, proper selection of the function characterizing the degree of repair at a given time, i.e. $\alpha(t)$ in one-dimensional and $\alpha_{1}(t)$ and $\alpha_{2}(t)$ in two-dimensional warranties, is essential. Since the expected warranty cost is highly sensitive to the repair policy, adoption of a poorly selected function may result in excessive cost to the manufacturer. The manufacturer usually has a chance to apply a sequence of progressively decreasing degrees of repair to a product. When there is a large number of alternative repair types/ degrees, the function can take an almost continuous form, a hypothetical example of which is to be considered in the computational analysis of Section 5.2.

\subsection{Modeling the expected number of failures under one- and two- dimensional warranty}

We consider the one-dimensional warranty and the most general two-dimensional warranty in which the warrantor agrees to repair or replace a failed item at no charge to the buyer up to a time limit $W$ or up to a usage limit $U$, whichever occurs first.

For the one-dimensional warranty, the expected number of failures within the warranty limit $W$ is as follows:

$M_{q}^{1}(W)=E[N(W)]=\sum_{n=0}^{\infty} n P(N(W)=n)=\sum_{n=1}^{\infty} F^{(n)}(W)$,

where $F^{(n)}(\cdot)$ is the $n$-fold convolution corresponding to the c.d.f. of $\sum_{i=1}^{n} T_{i}$. Since $Y_{n}$ 's are independent, $T_{n}$ 's are also independently but not identically distributed random variables. Therefore, we can write the joint density as the product of the marginal densities to obtain the $n$-fold convolution as follows:

$$
\begin{aligned}
F^{(n)}(t)= & P\left(T_{1}+T_{2}+\cdots+T_{n} \leqslant t\right) \\
= & \iiint_{t_{1}+t_{2}+\cdots+t_{n} \leqslant t} \cdots \int_{1} f_{1}\left(t_{1}\right) f_{2}\left(t_{2}\right) \cdots f_{n}\left(t_{n}\right) \mathrm{d} t_{n} \cdots \mathrm{d} t_{2} \mathrm{~d} t_{1} \\
= & \int_{t_{1}=0}^{t} \int_{t_{2}=0}^{t-t_{1}} \\
& \times \int_{t_{3}=0}^{t-t_{1}-t_{2}} \cdots \int_{t_{n}=0}^{t-\sum_{i=1}^{n-1}} t_{i} f_{1}\left(t_{1}\right) f_{2}\left(t_{2}\right) \cdots f_{n}\left(t_{n}\right) \mathrm{d} t_{n} \cdots \mathrm{d} t_{2} \mathrm{~d} t_{1} .
\end{aligned}
$$

Note that this $n$-fold convolution takes the following form for a static repair policy with a degree of repair denoted by $\alpha$

$$
\begin{aligned}
F^{(n)}(t)= & \int_{t_{1}=0}^{t} \int_{t_{2}=0}^{t-t_{1}} \\
& \times \int_{t_{3}=0}^{t-t_{1}-t_{2}} \ldots \int_{t_{n}=0}^{t-\sum_{i=1}^{n-1} t_{i}} f\left(t_{1}\right) \alpha^{-1} f\left(\alpha^{-1} t_{2}\right) \alpha^{-2} \\
& f\left(\alpha^{-2} t_{3}\right) \ldots \alpha^{1-n} f\left(\alpha^{1-n} t_{n}\right) \mathrm{d} t_{n} \ldots \mathrm{d} t_{2} \mathrm{~d} t_{1} .
\end{aligned}
$$

Under two-dimensional warranties, the manufacturer covers the cost of repair or replacement for failures that occur up to time limit $W$ and usage limit $U$. The warranty ceases either at time limit $W$ or at usage limit $U$ whichever occurs earlier. The expected number of failures over the warranty region $[0, W] \times[0, U]$ is expressed as

$$
\begin{aligned}
M_{q}^{2}(W, U) & =E[N(W, U)]=\sum_{n=0}^{\infty} n P(N(W, U)=n) \\
& =\sum_{n=1}^{\infty} F^{(n)}(W, U) .
\end{aligned}
$$

In the above equation, the general form of the $n$-fold convolution can be written as follows:

$$
\begin{aligned}
F^{(n)}(t, x)= & P\left(T_{1}+\cdots+T_{n} \leqslant t ; X_{1}+\cdots+X_{n} \leqslant x\right) \\
= & \iiint_{\substack{T_{1}+\cdots+T_{n} \leqslant t \\
X_{1}+\cdots+X_{2} \leqslant x}} \cdots f_{1}\left(t_{1}, x_{1}\right) \cdots f_{n}\left(t_{n}, x_{n}\right) \mathrm{d} x_{n} \mathrm{~d} t_{n} \cdots \mathrm{d} x_{1} \mathrm{~d} t_{1} \\
= & \int_{t_{1}=0}^{t} \int_{x_{1}=0}^{x} \cdots \int_{t_{n}=0}^{t-\sum_{i=1}^{n-1} t_{i}} \\
& \times \int_{x_{n}=0}^{x-\sum_{i=1}^{n-1} x_{i}} f_{1}\left(t_{1}, x_{1}\right) \cdots f_{n}\left(t_{n}, x_{n}\right) \mathrm{d} x_{n} \mathrm{~d} t_{n} \cdots \mathrm{d} x_{1} \mathrm{~d} t_{1} .
\end{aligned}
$$

\section{Application to a real life example}

This section presents application of the proposed approach to a problem faced by a leading beverage company that runs its own repair facilities for the industrial refrigerators used in its retail outlets in Turkey. We have access to the last five years' data collected by the Ankara repair facility that serves retail outlets located in 28 nearby cities. Failures are not covered under manufacturer's warranty; hence the company desires to keep its average repair costs under control by effectively managing the repair process. Failures occur mainly due to several major parts such as the compressor, the condenser, the evaporator, the thermostat and the door seals. Time until failure is affected by age as well as usage in terms of the number of times the internal temperature is disturbed by opening the door. Sales numbers at the retailer using a particular refrigerator constitute a reliable proxy to measure usage of that refrigerator.

The company performs three different kinds of repairs on failed refrigerators. In type 1 repair, only the part causing the failure is repaired or replaced depending on whichever is applicable to that specific part. In type 2 repair, after the leading cause of the failure is addressed, a preventive maintenance is carried out on the condenser, the most critical part affecting the lifetime. Finally, type 3 repair is an ultimate refurbishment operation in which all critical parts are checked or tested, and deteriorated parts are cleaned, repaired or replaced. Data indicates that repair types 1, 2 and 3 have average costs of, 10, 28 and 59 YTL (new Turkish Liras), respectively. Note that these costs correspond to the sum of the two variable cost components in the time and usage dimensions.

Statistical analyses were performed on a particular brand and model of refrigerator with data on 2150 refrigerators whose ages at the end of the five-year observation period varied between three and five. Of these, 285 failed at least once during the observation period. The correlation coefficient between time and usage is calculated as 0.976 . In view of this high correlation (close to unity), the remainder of the analysis is carried out solely based on the time dimension. Maximum likelihood estimation for right censored data yields that the time until first failure closely follows a Weibull distribution with shape and scale parameters of 1.68 and 158.24 , respectively. The resulting mean time until failure is 
138.59 months. A similar analysis of the 285 repaired refrigerators indicates that the time between the first and second failures is also Weibully distributed with the parameters listed in Table 1 for each repair type. Table 1 also shows the corresponding degree of repair for each repair type as the ratio of the mean time between the first and second failures to the mean time until the first failure.

Currently, the company applies the three repair types in a rather ad hoc manner. The proposed approach is used herein to help recommend a well-rounded, cost efficient repair policy. Time limits of the region within which a repair will be attempted are set in view of the distribution that characterizes the time until first failure. In particular, the limits are set systematically first at around a conservative value of 6 years and then at an upper extreme of 15 years. Three static repair policies with repair types 1 , 2 and 3 and a dynamic policy are tested. Each one of the static policies applies the corresponding repair type in any failure of the product. The dynamic policy in this particular application is defined as using repair type 2 in the first failure and repair type 1 in the succeeding failures. Since the product rarely fails more than twice within the imposed limits, the policy in effect achieves what the dynamic policy stated in a more idealistic fashion in Section 3.3.3 desires to accomplish.

Table 2 shows the expected number of failures and the corresponding repair cost under each policy both with a 6 -year limit and a 15-year limit. The best policy in terms of the expected repair cost is the static policy with repair type 1 . The dynamic policy outperforms the static policy with repair type 3 . The total repair costs with a 15-year limit are significantly smaller than the cost of a new refrigerator which is in the neighborhood of 400 YTL. Therefore, a reasonable recommendation for this company is to use a static repair policy with repair type 1 throughout the lifetime of a refrigerator.

A long term suggestion would be to also explore other types of repair to yield a larger number of alternatives in terms of the degree of repair and the corresponding cost. For example, the company may consider using different combinations of new and used replacement parts when addressing the cause of a failure. It may also consider replacing or maintaining other critical parts that do not necessarily have a direct impact on the current failure. An increase in the alternative repair types would allow a similar analysis considering a larger number of alternative static and dynamic policies. The new dynamic policies would be obtained

Table 1

Distribution parameters and degrees of repair for different repair types

\begin{tabular}{lll}
\hline Repair type & \multicolumn{2}{l}{ Time between failures } \\
\cline { 2 - 3 } & (shape, scale, $\left.\mu_{1}\right)$ & $\alpha_{1}$ \\
\hline 1 & $(1.55,38.38,34.51)$ & 0.25 \\
2 & $(1.02,127.57,126.54)$ & 0.91 \\
3 & $(1.11,140.51,135.18)$ & 0.98 \\
\hline
\end{tabular}

Table 2

Expected number of failures and expected repair cost under alternative policies

\begin{tabular}{llll}
\hline $\begin{array}{l}\text { Period } \\
\text { considered }\end{array}$ & $\begin{array}{l}\text { Repair } \\
\text { policy }\end{array}$ & $\begin{array}{l}\text { Expected number of } \\
\text { failures }\end{array}$ & $\begin{array}{l}\text { Expected repair } \\
\text { cost }\end{array}$ \\
\hline 6 years & Static 1 & 0.47497 & 4.74969 \\
& Static 2 & 0.25849 & 7.23778 \\
& Static 3 & 0.25650 & 15.1334 \\
& Dynamic & 0.37463 & 8.05844 \\
15 years & Static 1 & 2.37052 & 23.7052 \\
& Static 2 & 1.02638 & 28.7386 \\
& Static 3 & 0.99295 & 58.5839 \\
& Dynamic & 2.23448 & 35.3561 \\
\hline
\end{tabular}

by constructing different sequences of repair types at different times and/or counts of failure of a given product. Obviously, if a more reliable brand/model of a refrigerator becomes available, an improved policy such as the one described in Section 3.3.2 also becomes an option.

This practical example showed how the proposed approach can be applied to empirical data to formulate a preferred repair strategy. The next section analyzes a number of alternative static, improved and dynamic policies in a variety of settings in terms of product's reliability characteristics and structure of the function governing the unit repair cost.

\section{Computational experimentation}

This section presents computational results on the behavior of the expected warranty cost under various parameter settings in different types of policies. Reliability characteristics of the initial product are manipulated through the selection of the parameters of the Weibull and Normal distributions that are used to model the time/usage until first failure. Weibull distribution is widely used in reliability analysis due to its flexibility that allows accurate representation of a variety of lifetime distributions including lifetimes of products with multiple components. When the shape parameter exceeds one, the Weibull distribution has an increasing failure rate applicable to a multitude of products seen in practice. The Normal distribution is chosen in light of the empirical evidence indicating that items manufactured and tested under close control can be nicely modeled by its truncated versions (Davis, 1952). Products sold with warranty are expected to be manufactured under such close control. The need for truncation diminishes for practical purposes when the probability of obtaining a negative lifetime is sufficiently small.

Due to the analytical intractability of the infinite sum of series convolution corresponding to the expected number of failures in Eqs. (7), (8) and (10), a numerical method is used in the calculations. This method employs a recursive algorithm based on Composite Simpson's rule (Samatl, 2006). The algorithm starts out by applying Simpson's rule to the last integral, then for each evaluation point, the rule is applied to the second last integral and so on through the first integral at the beginning. Since the expected number of failures is stated in the form of an infinite sum, the process of numerical integration is truncated at the $n$th failure whose probability of occurrence is smaller than a given threshold level. This threshold level is set at 0.0001 in our experiments. The performance of the numerical method is tested with a univariate normal failure distribution, and it is seen that the difference between the numerical and analytical results is virtually zero.

In what follows, we present the experimental design for the computational study followed by the results for one- and twodimensional warranties.

\subsection{Experimental design}

The factors that we vary in the computational study consist of the reliability structure, the degree of repair, and the ratio between the fixed and variable components of the repair cost. We manipulate the reliability structure of the product by changing the mean time $\left(\mu_{1}\right)$ and mean usage $\left(\mu_{2}\right)$ to the first failure under univariate and bivariate Weibull and Normal distributions. If the ratio of the relevant mean to the corresponding warranty limit is larger than 1 , then the product is assumed to be highly reliable. On the other extreme, if this ratio is less than 0.5 , we then say that the product is unreliable. For other values of the ratio, the product has medium reliability. Considering that items sold with warranty are usually well-made, results for the highly reliable items are expected to 
have more practical significance. The degree of repair, $\alpha_{i}$, in the computational study takes on non-negative real values less than or equal to 1 where $\alpha_{i}=1$ corresponds to perfect repair (i.e., replacement). In the computational study, this parameter is varied between 0.5 and 1.0 for the static repair policy. For the improved policy, the improvement factor $\beta$ is set equal to 1.2 in each dimension, indicating a $20 \%$ improvement after the first failure, followed by a perfect repair strategy with $\alpha=1$. Finally, to investigate the effects of the cost function, the ratio of the fixed and variable cost components is varied $\left(c / c_{1}\right.$ in the univariate case, and $c / c_{1}$ and $c_{1} /$ $c_{2}$ in the bivariate case). The warranty period is assumed to be fixed for three time units in one-dimensional warranty policies, and 3 time and 3 usage units in two-dimensional policies.

\subsection{Computational results}

We first discuss the results with one-dimensional warranties followed by those with two-dimensional warranties.

\subsubsection{Results with one-dimensional warranties}

We systematically manipulate the shape and scale parameters of the Weibull, density to vary the reliability structure in terms of the mean time to the first failure. In particular, we fix the shape parameter $(\gamma)$ at 2 and vary the scale parameter $(\varphi)$ between 0.56 and 5.66 so as to obtain mean $\left(\mu_{1}\right)$ values between 0.5 and 5 . Since empirical evidence suggests that shape parameters in many applications are between 1 and 3.22, a shape parameter of 2 should be reasonable for the purposes of this investigation (Cohen and Whitten, 1988). Similarly, for the Normal distribution we change the reliability structure by varying the mean and variance. For each mean value, we assign the variance so as to maintain a coefficient of variation of $1 / 4$, which should be a reasonable assumption for the good quality items sold with warranty. In this way, we force the probability of realizing a negative inter-failure time to be negligible. With these two distributions, we cover products having both right-skewed and symmetric lifetime distributions and investigate different levels of reliability by changing the mean time to failure.

Table 3 displays the expected number of failures (Eq. (6)) and the corresponding cost values (Eq. (1)) for selected combinations of $\mu_{1}, \mathrm{c} / \mathrm{c}_{1}$ and $\alpha$ under various static policies. First few observations on these results are quite intuitive. For a given product reliability (i.e., $\mu_{1}$ ), a more thorough repair indicated by larger $\alpha$ values, results in a smaller expected number of failures. Similarly, when the repair strategy is fixed (i.e., given $\alpha$ ), more reliable products with larger $\mu_{1}$ values experience fewer failures on the average. For a product with $\mu_{1}=3$ under a Normal lifetime, the degree of repair has little effect on the number of failures. Although not shown in the table, the expected number of failures for a more reliable item with $\mu_{1}=5$ ranges between 0.256821 and 0.299703 under Weibull, and between 0.05498 and 0.055375 under Normal lifetimes. The effects of the factors on the expected cost are more interesting. The cost ratio $\left(c / c_{1}\right)$ has a significant effect on the expected cost. For instance, when $\mu_{1}=1$, the cost function decreases as $\alpha$ increases. Whereas when $\mu_{1}=3$ as $\alpha$ increases, the expected cost always increases for smaller cost ratios yet occasionally decreases for larger cost ratios. This effect is more pronounced under a Weibull lifetime due to the greater sensitivity of the number of failures to the degree of repair.

Tables 4 and 5 show the optimum degree of static repair corresponding to the minimum warranty cost (Eq. (1)) for different cost ratios and different mean times to the first failure under Weibull and Normal lifetime distributions. For an unreliable product with small mean time values, perfect repair is the selected repair type for any cost ratio. The reason for this is the significant impact of the degree of repair on the expected number of breakdowns, which more than compensates the corresponding increase in the repair cost. On the other hand, for a more reliable product with a larger mean time, a smaller degree of repair (smaller $\alpha$ ) gives the minimum cost when the fixed component of the cost is comparable with the variable component. However, if the fixed component is large, then a more extensive repair (larger $\alpha$ ) is needed to reduce the expected cost. In addition, for a product of medium reliability, the degree of repair varies as a function of the cost ratio. In particular, a more extensive repair is required as $c / c_{1}$ ratio increases for a given mean time to first failure. Eventually, when the fixed component hits a certain threshold, perfect repair becomes the most preferred option regardless of the initial product reliability.

Experimental results indicate that the improved policy offers no advantages in terms of the expected number of failures (Eq. (6)) over a perfect repair strategy for a highly reliable product with mean values around 5 . On the other hand, the improved policy is a desirable alternative for a less reliable product (e.g., $\mu_{1}=0.5$ ) as it results in a fewer number of expected failures. Table 6 shows the performance of the improved repair policy relative to the optimal static policy in terms of the expected cost (Eqs. (1) vs. (2)). Various $c / c_{1}$ ratios are considered. It is seen that if the fixed component of the cost is significantly larger than the variable component, then the improved repair policy dominates the optimum static policy for any given mean time to first failure under both Normal and Weibull lifetimes. This observation should not be surprising as a larger fixed cost component makes extensive repair more attractive also in the static policies. On the other hand, when the fixed cost is smaller than or comparable to the variable component, the improved policy tends to perform better than the optimum static policy for products of low and medium reliability. More specifically, for a given mean time to the first failure, the performance of the improved repair policy improves as the cost ratio increases,

Table 3

Expected number of failures and corresponding expected cost for various static policies

\begin{tabular}{|c|c|c|c|c|c|c|}
\hline \multirow[t]{2}{*}{$\alpha$} & \multicolumn{2}{|c|}{ Expected number of failures } & \multicolumn{2}{|c|}{ Expected cost $\left(c / c_{1}=0, c_{1}=10\right)$} & \multicolumn{2}{|c|}{ Expected cost $\left(c / c_{1}=10, c_{1}=10\right)$} \\
\hline & $\mu_{1}=1$ & $\mu_{1}=3$ & $\mu_{1}=1$ & $\mu_{1}=3$ & $\mu_{1}=1$ & $\mu_{1}=3$ \\
\hline \multicolumn{7}{|c|}{ Weibull lifetime } \\
\hline 1.00 & 2.60340 & 0.575055 & 26.0340 & 5.7506 & 286.3740 & 63.2561 \\
\hline 0.84 & 3.50697 & 0.602342 & 29.4585 & 4.9778 & 380.1555 & 65.2939 \\
\hline 0.68 & 5.48544 & 0.656892 & 37.3010 & 4.4669 & 585.8450 & 70.1561 \\
\hline 0.50 & $>8^{\mathrm{a}}$ & 0.850689 & $>40$ & 4.2534 & $>840$ & 89.3223 \\
\hline \multicolumn{7}{|c|}{ Normal lifetime } \\
\hline 1.00 & 2.56503 & 0.502253 & 25.6503 & 5.0225 & 282.1533 & 55.2478 \\
\hline 0.84 & 3.43516 & 0.503636 & 28.8553 & 4.2416 & 372.3713 & 54.7371 \\
\hline 0.68 & 6.66334 & 0.506150 & 45.3107 & 3.4839 & 711.6447 & 54.7171 \\
\hline 0.50 & $>8$ & 0.510925 & $>75$ & 2.7116 & $>1575$ & 56.9432 \\
\hline
\end{tabular}

${ }^{a}$ A lower bound provided to avoid excessive CPU time. 
Table 4

Optimum static repair degree for various first interarrival mean and cost ratios under a Weibull lifetime

\begin{tabular}{ccccc}
\hline $\begin{array}{c}\text { Scale } \\
\text { parameter }(\beta)\end{array}$ & $\mu_{1}$ & $c / c_{1}=0$ & $c / c_{1}=1$ & $c / c_{1}=10$ \\
\hline 0.56 & 0.50 & 1 & 1 & 1 \\
1.16 & 1.03 & 1 & 1 & 1 \\
1.76 & 1.56 & 0.88 & 1 & 1 \\
2.36 & 2.09 & 0.74 & 0.98 & 1 \\
2.96 & 2.62 & 0.64 & 0.84 & 1 \\
3.56 & 3.15 & 0.52 & 0.74 & 1 \\
4.16 & 3.69 & 0.50 & 0.66 & 1 \\
4.76 & 4.22 & 0.50 & 0.62 & 1 \\
5.36 & 4.75 & 0.50 & 0.58 & 1 \\
5.66 & 5.01 & 0.50 & 0.56 & 1 \\
\hline
\end{tabular}

Table 5

Optimum static repair degree for various first interarrival mean and cost ratios under a Normal lifetime

\begin{tabular}{c|ccccc}
\hline$\mu_{1}$ & $c / c_{1}=0$ & $c / c_{1}=1$ & $c / c_{1}=10$ & $c / c_{1}=100$ & $c / c_{1}=1000$ \\
\hline 0.5 & 1 & 1 & 1 & 1 & 1 \\
1.4 & 0.88 & 1 & 1 & 1 & 1 \\
2.3 & 0.5 & 0.68 & 1 & 1 & 1 \\
3.2 & 0.5 & 0.5 & 0.7 & 1 & 1 \\
4.1 & 0.5 & 0.5 & 0.58 & 0.86 & 1 \\
5.0 & 0.5 & 0.5 & 0.5 & 0.76 & 1 \\
\hline
\end{tabular}

and for a given cost ratio, it improves as the mean time to the first failure decreases.

Experimentation with the dynamic policy is performed with the degree of repair determined by the function $\alpha(t)=0.991+0.0093 t-$ $0.03 t^{2}$ selected idealistically as an example that displays the desired convexity characteristics. This hypothetical function is selected so that the degree of repair is almost perfect (i.e., $\alpha(0)=0.991$ ) at the beginning of the warranty period and reasonably good (i.e., $\alpha(3)=0.75$ ) at the end. In practice, manufacturers would have a chance to construct a function close to this form by

Table 6

Difference in expected cost between optimal static and improved repair policies

\begin{tabular}{|c|c|c|c|c|c|}
\hline$\beta$ & $\mu_{1}$ & $c / c_{1}=0(\%)$ & $c / c_{1}=5(\%)$ & $c / c_{1}=10(\%)$ & $c / c_{1}=100(\%)$ \\
\hline \multicolumn{6}{|c|}{ Weibull lifetime } \\
\hline 0.56 & 0.50 & 28.39 & 31.17 & 31.42 & 31.69 \\
\hline 1.16 & 1.03 & 10.13 & 16.52 & 17.10 & 17.72 \\
\hline 1.76 & 1.56 & -2.71 & 9.09 & 10.00 & 10.98 \\
\hline 2.36 & 2.09 & -19.28 & 4.63 & 5.76 & 6.98 \\
\hline 2.96 & 2.62 & -36.65 & 2.02 & 3.29 & 4.64 \\
\hline 3.56 & 3.15 & -54.50 & 0.47 & 1.81 & 3.24 \\
\hline 4.16 & 3.69 & -72.58 & -0.51 & 0.88 & 2.36 \\
\hline 4.76 & 4.22 & -86.60 & -1.35 & 0.26 & 1.77 \\
\hline 5.36 & 4.75 & -97.19 & -2.22 & -0.17 & 1.36 \\
\hline 5.66 & 5.01 & -107.19 & -5.89 & -3.55 & -2.01 \\
\hline$\underline{\mu_{1}}$ & $c / c_{1}=0(\%)$ & $c / c_{1}=1(\%)$ & $c / c_{1}=10(\%)$ & $c / c_{1}=100(\%)$ & $c / c_{1}=1000(\%)$ \\
\hline \multicolumn{6}{|c|}{ Normal lifetime } \\
\hline 0.5 & 37.06 & 38.25 & 39.23 & 39.42 & 39.44 \\
\hline 1.4 & 1.00 & 8.23 & 13.09 & 14.05 & 14.16 \\
\hline 2.0 & -40.77 & -7.05 & 2.32 & 3.82 & 3.99 \\
\hline 3.2 & -126.27 & -38.29 & -3.22 & -0.02 & 0.15 \\
\hline 4.1 & -134.92 & -43.57 & -4.69 & -0.18 & 0.04 \\
\hline 5.0 & -137.42 & -45.09 & -5.53 & -0.29 & 0.01 \\
\hline
\end{tabular}

considering a sequence of progressively decreasing degrees of repair. Fig. 1 shows the percentage difference in the expected cost relative to the optimum static policy (Eqs. (4) vs. (1)) as a function of the degree of repair for a Weibull lifetime. It is observed that the dynamic policy outperforms the optimum static policy when it is different from perfect repair. That is, the relative performance of the dynamic policy improves as the fixed component of the cost function decreases and the mean time to the first failure increases. On the other hand, for products with a normal failure distribution, Fig. 2 shows that for smaller ratios of the fixed to variable cost component, the dynamic policy performs better only when the product quality is either low or high. When the fixed cost component is much larger than the variable component, the dynamic policy always dominates the optimum static repair policy. The difference in the trends seen in Figs. 1 and 2 may be attributable to the greater sensitivity of the expected number of failures to the degree of repair under a Weibull distribution.

\subsubsection{Results with two-dimensional warranties}

Along the same lines in Section 5.2.1, we set the shape parameters of both the time and usage dimensions at 2 , and manipulate the two scale parameters to vary the mean time and usage until the first failure. Table 7 shows the effects of product reliability and the degree of repair on the expected number of breakdowns (Eq. (9)) with various static policies both under Weibull and Normal life-

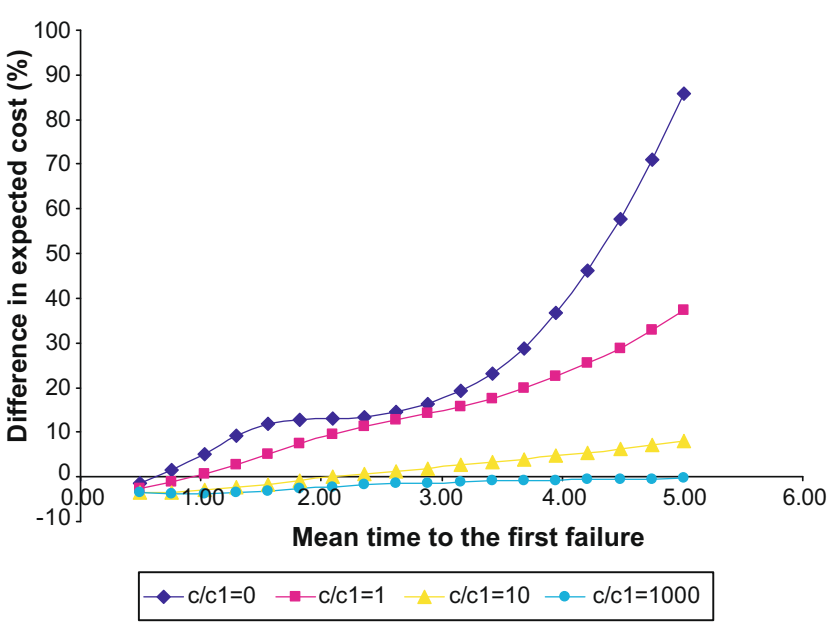

Fig. 1. Expected cost under dynamic policy relative to the optimum static policy under a Weibull lifetime.

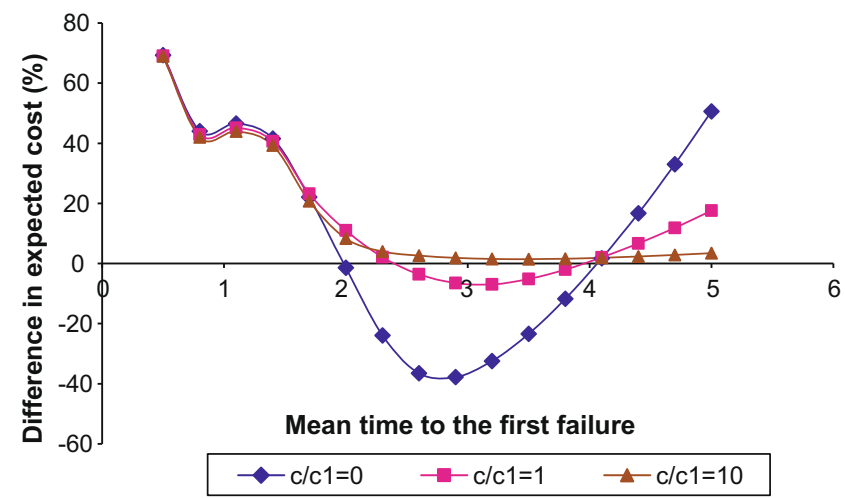

Fig. 2. Expected cost under dynamic policy relative to the optimum static policy under a Normal lifetime. 
Table 7

Expected number of failures under various static policies $(\rho=0.2)$

\begin{tabular}{|c|c|c|c|c|c|c|}
\hline \multirow[t]{2}{*}{$\left(\alpha_{1}, \alpha_{2}\right)$} & \multicolumn{3}{|l|}{$\mu_{1}=\mu_{2}$} & \multicolumn{3}{|l|}{$\underline{\mu_{1} \neq \mu_{2}}$} \\
\hline & $(5,5)$ & $(3,3)$ & $(1.5,1.5)$ & $(5,3)$ & $(5,1)$ & $(3,1)$ \\
\hline \multicolumn{7}{|l|}{ Weibull lifetime } \\
\hline$(1.0,1.0)$ & 0.090153 & 0.338829 & 1.15429 & 0.163960 & 0.257874 & 0.620392 \\
\hline$(1.0,0.8)$ & 0.090466 & 0.343277 & 1.22612 & 0.164710 & 0.258058 & 0.62301 \\
\hline$(1.0,0.5)$ & 0.091333 & 0.354343 & 1.34137 & 0.166356 & 0.258111 & $>0.82$ \\
\hline$(0.8,1.0)$ & 0.090466 & 0.343277 & 1.22612 & 0.165217 & 0.264394 & 0.659544 \\
\hline$(0.8,0.8)$ & 0.090917 & 0.349766 & 1.34158 & 0.166384 & 0.264819 & 0.666086 \\
\hline$(0.8,0.5)$ & 0.092233 & 0.367364 & $>1.35$ & 0.168995 & 0.265148 & $>0.82$ \\
\hline$(0.5,1.0)$ & 0.091333 & 0.354343 & 1.34137 & 0.169103 & 0.292529 & 0.817921 \\
\hline$(0.5,0.8)$ & 0.092233 & 0.367364 & $>1.35$ & 0.17247 & 0.295131 & $>0.82$ \\
\hline$(0.5,0.5)$ & 0.095038 & 0.416865 & $>1.35$ & 0.180836 & 0.296013 & $>0.82$ \\
\hline \multicolumn{7}{|l|}{ Normal lifetime } \\
\hline$(1.0,1.0)$ & 0.006120 & 0.282020 & 1.30157 & 0.035645 & 0.054791 & 0.502419 \\
\hline$(1.0,0.8)$ & 0.006120 & 0.282058 & 1.42095 & 0.036123 & 0.054793 & 0.504250 \\
\hline$(1.0,0.5)$ & 0.006120 & 0.282265 & 1.52067 & 0.036126 & 0.054810 & 0.504940 \\
\hline$(0.8,1.0)$ & 0.006120 & 0.282058 & 1.42095 & 0.036123 & 0.054840 & 0.506542 \\
\hline$(0.8,0.8)$ & 0.006120 & 0.282148 & 1.61625 & 0.036125 & 0.054847 & 0.506548 \\
\hline$(0.8,0.5)$ & 0.006120 & 0.282678 & 1.83357 & 0.036134 & 0.054849 & 0.506591 \\
\hline$(0.5,1.0)$ & 0.006120 & 0.282265 & 1.52067 & 0.036130 & 0.055404 & 0.541821 \\
\hline$(0.5,0.8)$ & 0.006120 & 0.282678 & 1.83357 & 0.036142 & 0.055510 & 0.543897 \\
\hline$(0.5,0.5)$ & 0.006123 & 0.285225 & $>1.84$ & 0.036208 & 0.055614 & 0.545218 \\
\hline
\end{tabular}

Table 8

Expected number of failures under various static policies with equal means and different correlation values

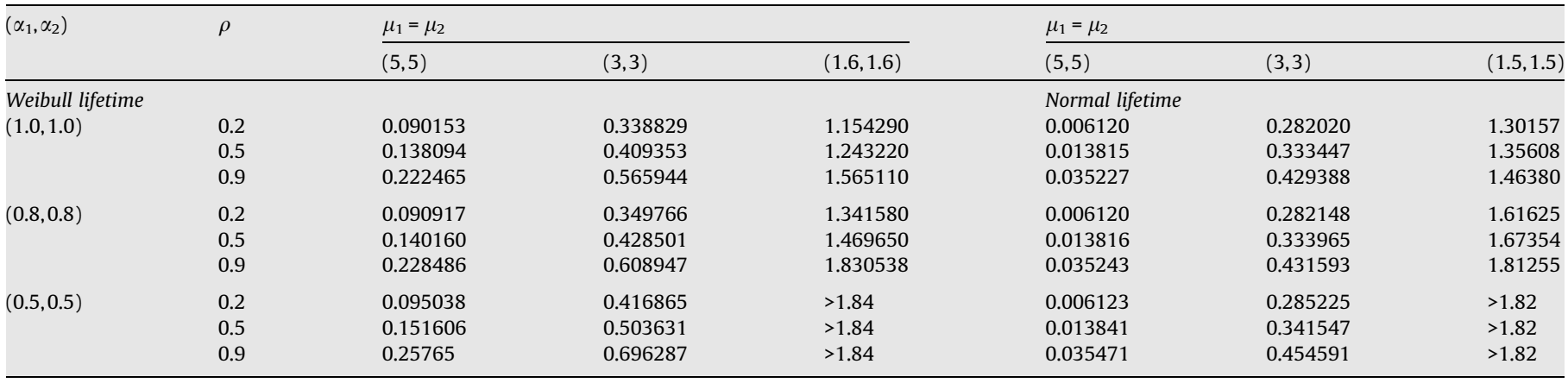

Table 9

Optimal degree combination for static repair $\left(c=c_{1}, \rho=0.2\right)$

\begin{tabular}{|c|c|c|c|c|c|c|c|}
\hline \multirow{2}{*}{$\frac{c=c_{1}}{c_{2}}$} & \multicolumn{3}{|l|}{$\mu_{1}=\mu_{2}$} & \multicolumn{4}{|l|}{$\mu_{1} \neq \mu_{2}$} \\
\hline & $(5,5)$ & $(3,3)$ & $(1.5,1.5)$ & $(5,3)$ & $(5,1)$ & $(3,1)$ & $(2,1.6)$ \\
\hline 0.1 & $\begin{array}{l}\text { Weibull lifetime } \\
(0.5,0.5)\end{array}$ & $(0.5,0.5)$ & $(1.0,0.5)$ & $(0.8,0.5)$ & $(0.8,0.5)$ & $(1.0,0.8)$ & $(1.0,0.8)$ \\
\hline 1 & $(0.5,0.5)$ & $(0.5,0.5)$ & $(1.0,0.5)$ & $(0.5,0.5)$ & $(0.5,0.5)$ & $(0.8,0.8)$ & $(1.0,0.8)$ \\
\hline 10 & $(0.5,0.8)$ & $(0.5,1.0)$ & $(0.5,1.0)$ & $(0.5,1.0)$ & $(0.5,0.5)$ & $(0.8,0.8)$ & $(0.5,1.0)$ \\
\hline 10000 & $(0.5,1.0)$ & $(0.5,1.0)$ & $(0.5,1.0)$ & $(0.5,1.0)$ & $(0.5,1.0)$ & $(0.8,1.0)$ & $(0.5,1.0)$ \\
\hline \multirow[t]{2}{*}{$\frac{c=c_{1}}{c_{2}}$} & \multicolumn{3}{|l|}{$\mu_{1}=\mu_{2}$} & \multicolumn{4}{|l|}{$\underline{\mu_{1}} \neq \mu_{2}$} \\
\hline & $(5,5)$ & $(3,3)$ & $(1.5,1.5)$ & $(5,3)$ & $(5,1)$ & $(3,1)$ & $(2,1.5)$ \\
\hline 0.1 & $\begin{array}{l}\text { Normal lifetime } \\
(0.5,0.5)\end{array}$ & $(0.5,0.5)$ & $(1.0,0.5)$ & $(0.5,0.5)$ & $(0.5,0.5)$ & $(0.5,0.5)$ & $(1.0,0.5)$ \\
\hline 1 & $(0.5,0.5)$ & $(0.5,0.5)$ & $(1.0,0.5)$ & $(0.5,0.5)$ & $(0.5,0.5)$ & $(0.5,0.5)$ & $(1.0,0.5)$ \\
\hline 10 & $(0.5,0.5)$ & $(0.5,0.5)$ & $(0.5,1.0)$ & $(0.5,0.5)$ & $(0.5,0.5)$ & $(0.5,0.5)$ & $(0.5,1.0)$ \\
\hline 10000 & $(0.5,1.0)$ & $(0.5,1.0)$ & $(0.5,1.0)$ & $(0.5,1.0)$ & $(0.5,1.0)$ & $(0.5,1.0)$ & $(0.5,1.0)$ \\
\hline
\end{tabular}

times. Results indicate that for a given mean vector $\left(\mu_{1}, \mu_{2}\right)$, the expected number of failures increases as the degree of repair decreases along at least one-dimension. In parallel with the results under one-dimensional warranties, when the product reliability deteriorates, the expected number of failures increases significantly.
Table 8 investigates the effect of the correlation coefficient $(\rho)$ varied as $0.2,0.5$ and 0.9 under various static policies. It is observed that as the time and usage dimensions become more dependent on each other, the expected number of failures (Eq. (9)) increases. Results suggest no significant interaction between the degree of repair $\left(\alpha_{1}, \alpha_{2}\right)$ and the correlation coefficient. 
Samatl (2006) observes that when the variable components of the cost function are equal to each other, the ratio of the fixed component to the variable components affects the selection of the optimal repair policy in a similar way as in one-dimensional warranty. Table 9 presents the optimal degree of static repair for different relative magnitudes of the two variable components. The fixed cost component is set equal to one of the variable components as $c=c_{1}$. Results indicate that the optimum degree of repair tends to be larger for the dimension with a lower variable cost component.

Table 10

Percentage difference in the expected cost under optimal static and improved repair policies $\left(c_{1}=c_{2}\right)$

\begin{tabular}{|c|c|c|c|c|c|c|c|}
\hline \multirow[t]{2}{*}{$\frac{c}{c_{1}=c_{2}}$} & \multirow[t]{2}{*}{$\rho$} & \multicolumn{3}{|l|}{$\mu_{1}=\mu_{2}$} & \multicolumn{3}{|l|}{$\mu_{1} \neq \mu_{2}$} \\
\hline & & $(5,5)$ & $(3,3)$ & $(1.6,1.6)$ & $(5,3)$ & $(5,1)$ & $(3,1)$ \\
\hline & & \multicolumn{3}{|c|}{ Weibull lifetime } & & & \\
\hline \multirow[t]{3}{*}{1} & 0.2 & -60.47 & -35.65 & -5.57 & -52.82 & -45.33 & -15.81 \\
\hline & 0.5 & -53.30 & -34.12 & -7.28 & -47.01 & -46.02 & -15.96 \\
\hline & 0.9 & -34.52 & -26.69 & -2.83 & -45.47 & -45.06 & -15.75 \\
\hline \multirow[t]{3}{*}{10} & 0.2 & -6.80 & -2.04 & 5.39 & -5.66 & -6.13 & -0.27 \\
\hline & 0.5 & -5.12 & -0.61 & 5.99 & -5.39 & -6.70 & -0.74 \\
\hline & 0.9 & -3.29 & 1.50 & 8.04 & -5.95 & -6.19 & -1.07 \\
\hline \multirow[t]{3}{*}{10000} & 0.2 & 0.40 & 1.53 & 7.92 & 1.16 & 1.56 & 3.90 \\
\hline & 0.5 & 0.79 & 2.35 & 8.37 & 1.56 & 1.37 & 3.80 \\
\hline & 0.9 & 1.48 & 3.75 & 10.39 & 1.41 & 1.48 & 3.98 \\
\hline \multirow[t]{2}{*}{$\frac{c}{c_{1}=c_{2}}$} & $\rho$ & \multicolumn{3}{|l|}{$\mu_{1}=\mu_{2}$} & \multicolumn{3}{|l|}{$\underline{\mu_{1} \neq \mu_{2}}$} \\
\hline & & $(5,5)$ & $(3,3)$ & $(1.5,1.5)$ & $(5,3)$ & $(5,1)$ & $(3,1)$ \\
\hline & & \multicolumn{3}{|c|}{ Normal lifetime } & & & \\
\hline \multirow[t]{3}{*}{1} & 0.2 & -55.23 & -58.18 & -0.79 & -60.63 & -66.07 & -55.23 \\
\hline & 0.5 & -57.62 & -57.64 & -4.21 & -63.70 & -66.19 & -55.47 \\
\hline & 0.9 & -59.35 & -54.12 & -11.84 & -66.11 & -66.46 & -55.35 \\
\hline \multirow[t]{3}{*}{10} & 0.2 & -2.93 & -4.89 & 10.52 & -6.99 & -11.09 & -8.31 \\
\hline & 0.5 & -4.52 & -4.53 & 10.72 & -9.25 & -11.17 & -8.31 \\
\hline & 0.9 & -5.66 & -4.17 & 10.97 & -11.11 & -11.32 & -8.24 \\
\hline \multirow[t]{3}{*}{10000} & 0.2 & 8.64 & 5.89 & 13.41 & 3.43 & 0.01 & 0.26 \\
\hline & 0.5 & 7.10 & 5.01 & 13.60 & 2.24 & -0.02 & 0.26 \\
\hline & 0.9 & 5.61 & 4.02 & 13.84 & 0.02 & -0.02 & 0.26 \\
\hline
\end{tabular}

Table 11

Percentage difference in the expected cost under optimal static and dynamic repair policies $\left(c_{1}=c_{2}\right)$

\begin{tabular}{|c|c|c|c|c|c|c|c|}
\hline \multirow[t]{2}{*}{$\frac{c}{c_{1}=c_{2}}$} & \multirow[t]{2}{*}{$\rho$} & \multicolumn{3}{|c|}{$\mu_{1}=\mu_{2}$} & \multicolumn{3}{|c|}{$\underline{\mu_{1}} \neq \mu_{2}$} \\
\hline & & $(5,5)$ & $(3,3)$ & $(1.6,1.6)$ & $(5,3)$ & $(5,1)$ & $(3,1)$ \\
\hline & & \multicolumn{6}{|c|}{ Weibull lifetime } \\
\hline \multirow[t]{3}{*}{1} & 0.2 & 45.49 & 9.07 & 8.08 & 49.87 & 53.71 & 36.47 \\
\hline & 0.5 & 47.53 & 11.80 & 6.51 & 51.55 & 53.53 & 36.54 \\
\hline & 0.9 & 50.37 & 12.74 & 8.94 & 52.14 & 53.99 & 36.41 \\
\hline \multirow[t]{3}{*}{10} & 0.2 & 14.45 & 7.91 & 5.44 & 15.92 & 15.57 & 11.86 \\
\hline & 0.5 & 15.07 & 8.44 & 5.78 & 15.69 & 15.19 & 11.57 \\
\hline & 0.9 & 14.33 & 6.46 & 6.34 & 15.32 & 15.88 & 10.66 \\
\hline \multirow[t]{3}{*}{10000} & 0.2 & 2.90 & 2.87 & 3.59 & 3.49 & 3.96 & 3.67 \\
\hline & 0.5 & 2.45 & 2.71 & 3.76 & 3.43 & 4.01 & 3.69 \\
\hline & 0.9 & 2.37 & 2.70 & 4.43 & 3.80 & 4.37 & 3.16 \\
\hline \multirow[t]{2}{*}{$\frac{c}{c_{1}=c_{2}}$} & \multirow[t]{2}{*}{$\rho$} & \multicolumn{3}{|l|}{$\underline{\mu_{1}=\mu_{2}}$} & \multicolumn{3}{|c|}{$\mu_{1} \neq \mu_{2}$} \\
\hline & & $(5,5)$ & $(3,3)$ & $(1.5,1.5)$ & $(5,3)$ & $(5,1)$ & $(3,1)$ \\
\hline & & \multicolumn{6}{|c|}{ Normal lifetime } \\
\hline \multirow[t]{3}{*}{1} & 0.2 & 31.07 & -15.48 & -1.60 & 36.38 & 41.68 & 14.36 \\
\hline & 0.5 & 30.10 & -15.10 & -1.24 & 36.56 & 41.60 & 14.20 \\
\hline & 0.9 & 29.42 & -12.56 & -7.75 & 37.24 & 43.77 & 15.07 \\
\hline \multirow[t]{3}{*}{10} & 0.2 & 11.97 & 2.87 & -1.02 & 13.80 & 15.73 & 13.25 \\
\hline & 0.5 & 10.78 & 3.15 & -0.57 & 14.03 & 15.61 & 11.71 \\
\hline & 0.9 & 9.96 & 3.34 & -0.07 & 14.93 & 19.66 & 14.68 \\
\hline \multirow[t]{3}{*}{10000} & 0.2 & 7.69 & 5.89 & -2.76 & 7.35 & 8.98 & 6.00 \\
\hline & 0.5 & 6.32 & 4.96 & -2.38 & 8.46 & 8.90 & 5.95 \\
\hline & 0.9 & 5.00 & 3.78 & -1.88 & 9.00 & 10.93 & 7.67 \\
\hline
\end{tabular}

As in one-dimensional warranty, the improved policy for a reliable product offers no advantages over a perfect repair in terms of the expected number of failures. Table 10 analyzes the performance of the improved policy relative to the optimum static policy in terms of the expected cost (Eqs. (1) vs. (3)). When the fixed component of the cost function is significantly larger than the variable components, the improved policy generally dominates the optimum static policy, which turns out to be perfect repair.

For the two-dimensional dynamic policy, the degree of repair for the time dimension is determined by the same function as in the one-dimensional case and the degree of repair for the usage dimension is calculated such that $\alpha_{2, i}=\alpha_{1}\left(\sum_{k=1}^{i-1} T_{k}\right) \frac{\mu_{2}}{\mu_{1}}$. Table 11 shows the percentage difference between the dynamic and optimal static policies (Eqs. (1) vs. (5)) where a negative sign indicates that the cost of optimal static policy is smaller than that of dynamic. The relative performance of the dynamic policy improves under a Weibull lifetime when the fixed component of the cost decreases. Performance of the dynamic policy under a Normal lifetime displays an analogous behavior to that observed in Fig. 2 in the single-dimensional analysis.

\section{Conclusion}

Computational results show that the dynamic policy generally outperforms both static and improved policies on highly reliable products, whereas the improved policy is the best performer for products with low reliability. Although, the increasing number of factors arising in the analysis of two-dimensional policies renders generalizations difficult, several insights are offered for the selection of the rectification action based on empirical evidence.

As a future direction, the analysis can be extended to multidimensional warranties. For example, a three-dimensional quasirenewal process may be used to model the warranty policy offered for the flight engines. In addition, the study can be generalized to accommodate multi-component systems. In this case, each component failure process may be modeled as a quasi-renewal process.

\section{Acknowledgement}

The authors would like to thank the two anonymous referees for their helpful suggestions and constructive comments on an earlier version of this manuscript.

\section{References}

Bai, J., Pham, H., 2005. Repair-limit risk-free warranty policies with imperfect repair. IEEE Transactions on Systems, Man and Cybernetics, Part A 35 (6), 765-772.

Barlow, R., Hunter, L., 1960. Optimum preventive maintenance policies. Operations Research 8, 90-100.

Boland, P.J., Proschan, F., 1982. Periodic replacement with increasing minimal repair costs at failure. Operations Research 30 (6), 1183-1189.

Choi, C.H., Yun, W.Y., 2006. A repair cost limit policies with fixed warranty period. In: Yun, W.Y., Dohi, T. (Eds.), Advanced reliability modeling II: reliability testing and improvement: Proceedings of the Second International Workshop Aiwarm 2006. World Scientific Publishing Co. Pte. Ltd., Singapore, 2006, pp. 353-360.

Chukova, S., Johnston, M.R., 2006. Two-dimensional warranty repair strategy based on minimal and complete repairs. Mathematical and Computer Modeling 44, 1133-1143.

Chukova, S., Hayakawa, Y., Johnston, M.R., 2006. Two-dimensional warranty: Minimal/complete repair strategy. In: Yun, W.Y., Dohi, T. (Eds.), Advanced reliability modeling II: Reliability testing and improvement: Proceedings of the Second International Workshop Aiwarm 2006. World Scientific Publishing Co. Pte. Ltd., Singapore, 2006, pp. 361-368.

Cleroux, R., Dubuc, S., Tilquin, C., 1979. The age replacement with minimal repair and random repair costs. Operations Research 27 (6), 1158-1167.

Cohen, A.C., Whitten, B.J., 1988. Parameter Estimation in Reliability and Life Span Models. Marcel Dekker Inc., New York and Basel.

Dagpunar, J.S., 1997. Renewal-type equations for a general repair process. Quality and Reliability Engineering International 13, 235-245. 
Davis, D.J., 1952. An analysis of some failure data. Journal of the American Statistical Association 47, 113-150.

Dimitrov, B., Chukova, S., Khalil, Z., 2004. Warranty costs: An age-dependent failure/ repair model. Naval Research Logistics 51 (7), 959-976.

Iskandar, B.P., Murthy, D.N.P., 2003. Repair-replace strategies for two-dimensiona warranty policies. Mathematical and Computing Modeling 38, 1233-1241.

Iskandar, B.P., Murthy, D.N.P., Jack, N., 2005. A new repair-replace strategy for items sold with a two-dimensional warranty. Computers and Operations Research 32, 669-682.

Karim, M.R., Suzuki, K., 2005. Analysis of warranty claim data: A literature review. International Journal of Quality and Reliability Management 22 (7), 667-686.

Kijima, M., 1989. Some results for repairable systems with general repair. Journal of Applied Probability 26, 89-102.

Murthy, D.N.P., Blischke, W.R., 1992a. Product warranty management - II: An integrated framework for study. European Journal of Operational Research 62 261-281.

Murthy, D.N.P., Blischke, W.R., 1992b. Product warranty management - III: A review of mathematical models. European Journal of Operational Research 63, 1-34.
Murthy, D.N.P., Djamaludin, I., 2002. New product warranty: A literature review. International Journal of Production Economics 79, 231-260.

Nguyen, D.G., Murthy, D.N.P., 1984. A combined block and repair limit replacement policy. Journal of the Operational Research Society 35 (7), 653-658.

Phelps, R.I., 1983. Optimal policy for minimal repair. Journal of the Operational Research Society 34 (5), 425-427.

Samatl, G., 2006. Warranty Cost Analysis Under Imperfect Repair. Unpublished Masters Thesis, Bilkent University, Ankara, Turkey.

Thomas, M.U., Rao, S.S., 1999. Warranty economic decision models: A summary and some suggested directions for future research. Operations Research 47 (6), 807820 .

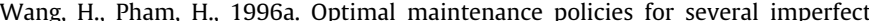
repair models. International Journal of Systems Science 27 (6), 543-549.

Wang, H., Pham, H., 1996b. A quasi renewal process and its applications in imperfect maintenance. International Journal of Systems Science 27 (10), 10551062.

Yanez, M., Joglar, F., Modarres, M., 2002. Generalized renewal process for analysis of repairable systems with limited failure experience. Reliability Engineering and System Safety 77, 167-180. 